\title{
Efficient Distributed Vulnerability Assessment by Utilizing Miniaturized Computers
}

\author{
Yi Hu${ }^{1}$, Dustin Sulek ${ }^{1}$, Anthony Carella ${ }^{1}$, Joshua Cox ${ }^{1}$, Allyson Frame ${ }^{1}$, Karen Cipriano ${ }^{1}$, \\ Hong-xia Wang ${ }^{2}$ \\ Northern Kentucky University, USA ${ }^{1}$ \\ Southwest Jiaotong University, China ${ }^{2}$
}

\begin{abstract}
Vulnerability assessment is the practice of testing a computer system, network or application to identify, measure and rank vulnerabilities within the system. This project's goal is to create a distributed vulnerability assessment architecture utilizing multiple miniaturized computers such as Raspberry Pi 2 Model B. The scheme delegates scanning tasks to low-cost and self-contained miniaturized devices for pursuing vulnerability assessment to achieve load balancing and enhanced performance. A cloud based dashboard application allows each miniaturized computer to register itself and controls the vulnerability assessment process for multiple networks. This allows the security professional to conduct assessment process and view vulnerability reports remotely. This paper describes the detailed design and implementation of the proposed architecture. In addition, different load balancing schemes for enhancing the performance of the vulnerability assessment are proposed and compared. The performance and assessment results prove the viability of automated distributed vulnerability assessment using miniaturized computers.
\end{abstract}

\section{Introduction}

Computer security is at odds with business. In business, decisions are made based on whether they produce profits or save the company money. After all, profits are the driving force behind every company's ability to stay in business. It is this process that drives computer security to the fringes of acceptance among businesses. Many companies don't understand the risk they face until it's too late, forcing computer security related decisions to be reactionary

Companies, both large and small, are required to store their data in some manner. Whether it is an inhouse data center or outsourced storage, data is always at risk of being compromised. Unwanted malicious attackers who look for and exploit vulnerable computer systems are constantly bombarding IT infrastructures to attempt to steal data. When hackers are successful at infiltrating a company or organization network and steal their data, the result is often an expensive and resource intensive restoration process. A company can often lose business over a data breach.
Data is the crown jewel of every single organization. It can be anything from a test grade, a list of your purchases at a grocery store to your social security number. Therefore, a security breach resulting in information loss not only affects the companies, but also its customers, employees, and in some cases non-customers - in other words it could affect you. This can result in financial and personal loss. With technology infiltrating every aspect of our businesses and personal lives, it is crucial that information assurance and securing computer systems and networks is a top priority for companies, organizations, and developers alike. Interestingly enough, this is often not the case. Network assessments and subsequence changes are costly, resource intensive, and if outsourced, may include security vulnerabilities of its own.

This project aims at developing a distributed vulnerability assessment architecture by employing low-cost and self-contained miniaturized computers for vulnerability assessment. The miniaturized computers employed are Raspberry Pi 2 Model B. The distributed architecture delegates certain sections of the network to each respective Raspberry $\mathrm{Pi}$, allowing for maximum utilization of the hardware. The vulnerability assessment process utilizes OpenVAS [1], an open source vulnerability scanner. Through the use of customized Python scripts, the vulnerability scanning processes are automated as much as possible. Using the Metasploit database, Python and PHP scripts, the assessment results are presented in a user friendly and accessible manner through an AWS EC2 dashboard application.

\section{Motivation for distributed vulnerability assessment using miniaturized computers}

As stated above, vulnerability assessment is the practice of testing a computer system, network, or application to identify, measure and rank vulnerabilities within the system. Hackers will often perform the same assessment that a security analyst would to search for specific vulnerabilities that they can exploit to gain access to a company network or applications. Therefore, it is important that companies stay on top of their assessments to 
remove their infrastructure and software vulnerabilities.

There are various methodologies and guidelines $[2,3,4,5,6,7,8,9]$ for performing penetration testing and vulnerability assessment. Some small standalone devices exist for penetration testing such as Pwn Plug R3 [10], MinPwner [11], WiFi Pineapple [12]. To the best of our knowledge, no research has been performed on utilizing a network of miniaturized computers for performing distributed penetration testing. The most related research works are on mobile vulnerability assessment, penetration testing using traditional PCs or standalone miniaturized devices, and attack vectors of miniaturized computers.

\section{Design of distributed vulnerability assessment}

\subsection{Distributed vulnerability assessment architecture}

The miniaturized computers, or pentest boxes, are pre-planted in each network to be scanned for vulnerability assessment. Figure 1 is a representation of how our nodes are set up in the architecture.

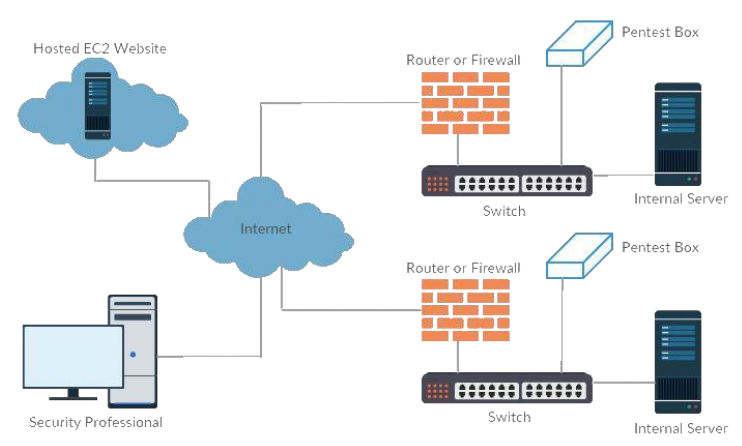

Figure 1. Architecture of distributed vulnerability assessment

As you can see, our miniaturized computer (labeled Pentest Box in Figure 1) is placed behind the router/firewall and has immediate access to the server to be scanned (Note: There could be multiple internal computers on a network. For simplicity reason, we only draw one.). The pentest box connects out to a remote AWS EC2 instance through reverse SSH connection since most firewalls filter incoming SSH connections but not outgoing $\mathrm{SSH}$ connections. This also allows for remote control of each pentest box through the remote EC2 dashboard. To create a distributed assessment system multiple pentest boxes are placed on separate networks and each has delegated tasks based on its location. This provides a window into multiple networks from a single access point, mainly the dashboard application.
Below is the sequence of operations employed to connect each individual pentest box to the dashboard application and for the dashboard application to start the vulnerability scanning process.

1) Each pentest box connects to the remote AWS EC2 dashboard instance over a reverse SSH tunnel.

2) The pentest user can start live host scans by controlling each pentest box from the web interface of the dashboard.

3) The pentest user can then initiate vulnerability scans on selected live nodes via the dashboard.

4) Each pentest box sends a report back to the dashboard containing information about the vulnerabilities found.

\subsection{Building a self-contained vulnerability assessment device}

Our first goal is to build a self-contained vulnerability assessment device before implementing a distributed penetration testing framework. As it turns out it would also be the most time consuming of the goals. Miniaturized computing is a new field in terms of consumer devices, resulting in a lack of software compatibility and documentation on the software/hardware relationships.

We decided to use a Raspberry Pi 2 Model B for our build. The Raspberry Pi 2 Model B was the newest edition in the Raspberry Pi line at the time of the experiment and has a $900 \mathrm{MHz}$ quad-core ARM Cortex-A7 CPU. Being ARM based, the Raspberry Pi has compatibility with many operating systems, including Kali Linux. We decided to use Kali Linux for its reputation as a digital forensics and penetration testing distribution. However, being ARM based also has its drawbacks. The default ARM distribution of Kali Linux is a bare bones version and does not come stock with many of the industry standard tools, including OpenVAS. As it turns out, OpenVAS was not very compatible with the Raspberry Pi and took weeks of tweaking to get a viable test version running on a device.

Many of the tools used in this project were installed from source and modified in order to correctly perform their tasks on the Raspberry Pi. Once the device was configured to the needs of the project, an image was made along with a README file documenting the steps taken for configuration and steps needed to customize the configuration to your needs. Interested readers may refer to URLs in these references [13, 14] for more information. This boilerplate image will allow for easier installation onto other devices as well as a means for fresh installation in the instance that any problems occur, such as a corrupted file system. 


\subsection{Vulnerability assessment}

The phase of vulnerability assessment employs OpenVAS which initiates the scan and is able to identify vulnerable OS, applications, network services, and possible backdoors. Although there exist other professional vulnerability scanners such as Tenable Nessus [15], Nexpose [16], Core Impact [17], Canvas [18], OpenVAS can be easily integrated into our project due to its open source nature.

After completing a scan, OpenVAS produces a report that comes in several different formats, including PDF and XML. Using Metasploit, an open source penetration testing framework, the data is imported from OpenVAS into the Metasploit PostgreSQL database. Once in Metasploit's PostgreSQL database, a Python script is utilized to upload the data from the database to our AWS EC2 dashboard application for generating distributed vulnerability assessment reports.

\subsection{AWS EC2 dashboard for distributed vulnerability assessment}

A dashboard application was developed to control multiple Raspberry Pi's for distributed vulnerability assessment. The dashboard is run from an AWS EC2 instance that is running an Apache 2 web server and is fully configured to listen for Raspberry Pi's which are attempting to make a connection to the instance. The instance is created using an AWS CloudFormation template which allows for a remote server to be created within minutes and removes human error during its setup and configuration.

To automate the vulnerability assessment process, each Raspberry $\mathrm{Pi}$ based pentest box registers itself to the EC2 dashboard application upon plugged into a network. This registration is established through the use of a reverse SSH tunnel which allows access to the Pi's network even behind a firewall. Once the pentest box registers with the dashboard, a new Pi icon is illustrated at the left side of the dashboard as depicted in Figure 2.

\subsection{Service and scanning automation design}

Automation of the assessment process contains multiple phases. The design of each automation process is explained in the following sections.

\subsubsection{Service Automation}

Firstly, we need to have required processes to start up upon boot. This is achieved through a simple Python script, titled startup.py. This script requires some information to run properly in which it can detect all the necessary information to run except for the EC2 instance's host name or IP address.

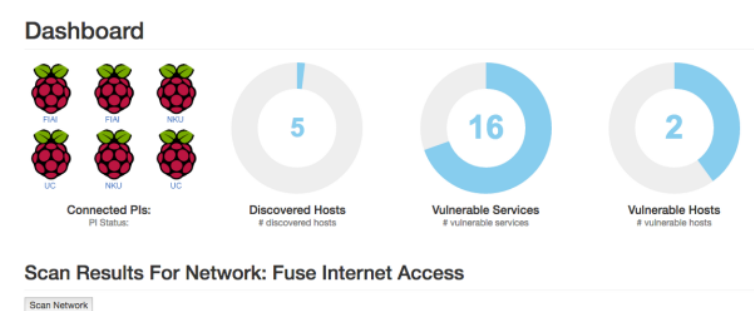

Figure 2. Dashboard depicting multiple registered Pi's for performing vulnerability assessment

For this reason, the address of the EC2 instance is made known to each $\mathrm{Pi}$. So it can register itself and establish a connection to the EC2 dashboard application. Beyond this, the startup script determines the IP address of the Raspberry Pi and the subnet mask that it uses, establishes tunnel connections to the postgreSQL database on port 5432 and creates an SSH tunnel over port 22 on the Pi, starts all OpenVAS services and runs a Metaspolit network scan. Services such as OpenVAS and Metasploit are required to be running because most of the project revolves around them to function correctly. Metasploit Framework and the PostgreSQL database were used to import the scan data into the dashboard. To ensure that this script runs every time the Raspberry Pi boots, the rc.local script contains the following lines of code:

- $\quad$ whois $\$($ dig + short myip.opendns.com @ resolverl.opendns.com) > /tmp/netInfo.tmp

- $\quad$ python2 letc/startup.py

The whois command gathers an abundance of information about the network that it is hidden in and writes this out to a temporary file called netInfo.tmp that a Python script on the EC2 instance will query. The second command tells the startup.py script to run. A symbolic link to the rc.local script also exists within the /etc/rc1.d directory which causes the rc.local script to run automatically each time the device boots.

\subsubsection{Scanning automation}

After we were able to automate the startup process for the services, we needed to automate the scanning process itself. The process however begins before the startup.py script ever runs. It begins with the configurations of the Raspberry Pi so that it has the ability to seamlessly and autonomously communicate with the Amazon EC2 server. A script called configureRasp.sh is run after installing Kali, Metasploit and OpenVAS. It starts by installing the autossh, whois and dnsutils packages which allow for connections to be made to the EC2 instance and provide details about the network in which the $\mathrm{Pi}$ is located. The script then updates the sshd_config file 
allowing for password authentication and an authorized keys file to allow for requests coming back from the server to run necessary commands to provide the user with more information. Finally, this script provides the proper public and private keys for the server and the $\mathrm{Pi}$, updates the pg_hba.conf file (Metasploit Postgresql database configuration file) to allow for password authentication and adds several lines to the rc.local file as described in the previous section.

A script called determineConnections.py was written for the server to gather all established tunnel connections to be used by the dashboard. A range from 29000 to 29999 is used to allow for ports in that range to connect to the PostgreSQL database. This leaves ports 30000 to 30999 (a Pi using 29000 would also use 30000) open for use of running commands and logging into each Raspberry Pi. The determineConnections.py script uses the netstat $-l$ command to gather connected ports and put them in a file called ssshp. Along with ports that are connected, the script comma delimits other information about each connected Raspberry Pi and writes this information to the ssshp file in the following form:

- Connected port, Organization ID, Organization Name, Running Scan

The Organization ID and Organization Name portions of the CSV file is data that is collected from the whois command that gets run from the rc.local script on Raspberry Pi startup. The determineConnections.py script completes by clearing the ssshp.tmp file that the dashboard uses to look for requested scans that the ssshp file has yet to learn about.

When you first access the dashboard application, you are shown a "Scan Network" button, as seen in Figure 2. When clicked, this button calls a Python script penScanCall.py. This script controls the scan that will run on the selected Raspberry Pi based on which button is clicked on the dashboard. When called using this button, this script will use Metasploit's version of nmap to scan your network for connected devices, and return their IP addresses in a table format. Once this list of IP addresses is returned they are all clickable links. Clicking on one of these links will direct the user to the details.php page of the website which contains more information and the button to scan for vulnerabilities of that device. From the details.php page a button exists that is labeled "Scan Vulernabilities" which calls the penScanCall.py script to run an OpenVAS vulnerability scan of the selected device from the Raspberry Pi. Following paragraphs describe how the penScanCall.py script runs an OpenVAS scan (please note the following are pertinent code segments and not the entire script).
OpenVAS contains the OpenVAS management protocol (OMP), which enables the use of XML based requests utilizing the full capabilities of OpenVAS. This protocol was utilized in our script for the automation of the scanning process.

a) The script will first authorize the user and call variables into action. The IP address that is clicked on will be saved in a variable "ip" and used to create a target. The code for target creation is as follows:

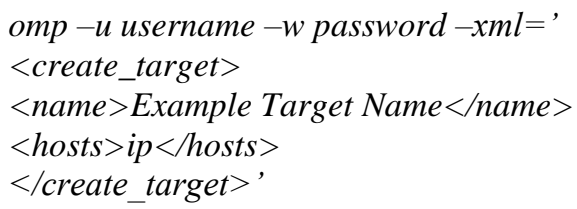

This segment will then produce a target id that will be saved under variable "target_id".

b) OpenVAS has multiple scan configurations that you can use. However, we chose to stick with one configuration titled "Full and Fast" which provides a good scan without overwhelming the Raspberry Pi. We will save the Scan Configuration ID under the variable scan_type.

c) Next we will create a task using the Target_ID and Scan_Config_ID. The code for scan creation is as follows:

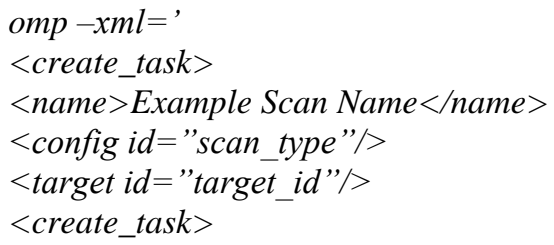

This segment will then produce a task ID that will be saved in the variable "task_id".

d) After we have obtained a task ID, we will start the scanning process using the following segment of code.

omp $-x m l=<$ start_task task_id="task_id" $/>$

Stop, pause and resume functionalities were added into the penScanCall.py script so that the user could have more control over the scans that they are running. The omp commands to stop and resume a scan are as follows:

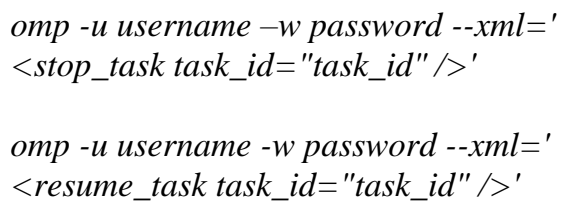

When the "Full and Fast" scan has completed, its scan status will become "Done" which tells the script to continue. The report id will be put into the variable "report_id" and creates a temporary file 
called openvas_scan.xml using the following command code:

omp - u username -w password $-i X$

'<get_tasks task_id="task_id" />'

omp - u username - w password $--x m l='$

$<$ get_reports report_id="report_id" format="XML" $1>$

$>$ openvas_scan.xml

The script ends by deleting the task_id, target_id and report_id from OpenVAS and imports the report data contained within the openvas_scan.xml file into the Metasploit database using the following command:

msfconsole $-q-x$ 'db_connect

msf3:msf3@localhost/msf3; db_import

openvas_scan.xml; exit $-y^{\prime}$

\section{Experiments and results}

The following sub-sections provide details of our experiment results. They also highlight the procedures taken for each goal and tools utilized to achieve it. The performance and assessment monitoring results are also illustrated.

\subsection{Vulnerability assessment report generation}

When the "Scan Network" button in Figure 2 is clicked, a Python script is initiated to perform a live host scan, i.e., ping scan, that finds all connected devices on the currently selected Raspberry Pi's network, including their IP addresses and operating systems. The results will look similar to Figure 3.

\begin{tabular}{ll}
\hline IP Address & Operating System \\
\hline 192.168 .200 .174 & Windows 2003 \\
\hline 192.168 .200 .52 & Windows XP \\
\hline 192.168 .200 .244 & Linux \\
\hline
\end{tabular}

Figure 3. Results of a live host scan

Individual IP addresses in Figure 3 are clickable and once clicked will direct the user to start a vulnerability scan using OpenVAS. This can be done remotely from the dashboard web application. The wait time is dependent on the number of live hosts and the number of running services on each host on the network. Once complete the dashboard will illustrate the results of the scan and the detailed nature of each vulnerability.

There are three reports on the dashboard that outline information gathered from a Raspberry Pi's Metasploit database. The first of these reports contains information about the IP address of each device on the selected Pi's network, the operating system of the device, the number of services that the device offers and the number of vulnerabilities the device has. Figure 4 shows vulnerabilities discovered on 3 testing systems.

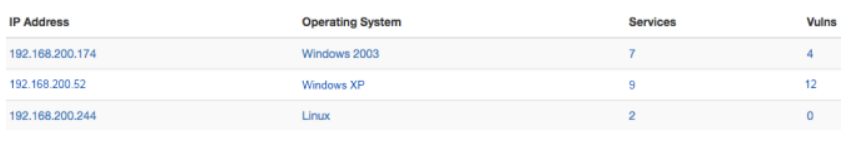

Figure 4. Vulnerabilities discovered on testing systems

The second report provides the user with information such as the active ports, protocols, the state of each port, and information outlining what is running on the ports. Figure 5 shows a sample port scanning result.

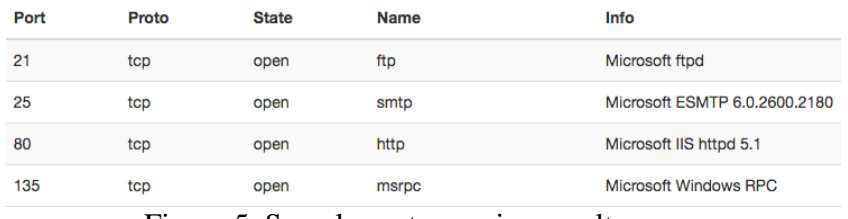

Figure 5. Sample port scanning result

The third report on the dashboard shows the name of each vulnerability as well as any existing information on the vulnerability which may include recommendations on how to address it. Figure 6 shows the details of vulnerabilities discovered.

\subsection{Monitoring vulnerability assessment}

Although the speed of vulnerability assessment using Raspberry Pi is not comparable to a dedicated $\mathrm{PC}$, we are curious to see whether running OpenVAS overwhelms the Pi. So we monitored the device when it performed the scan. We achieved this though a Python script that monitors the temperature, CPU usage, and memory usage of the device. Figure 7 illustrates the testing result. It can be seen that the CPU usage is somewhere between $10 \%$ to $90 \%$ although for most time CPU usage is below $50 \%$. The average CPU temperature is about 40 Celsius degrees which is considered normal under the load of vulnerability assessment.

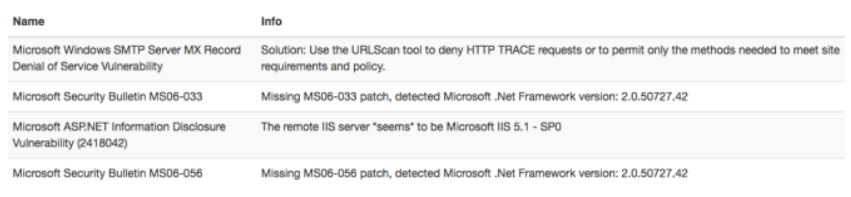

Figure 6. Details of vulnerabilities discovered

\subsection{Load balancing architecture}

All of the Raspberry Pi's purchased for the project are loaded with the same operating system and needed software. The load for assessing vulnerabilities in multiple nodes is distributed to 
individual Pi's. The automation scripts provide a foundation that can be built upon, allowing pen testing devices to be set up in the same network or segregated ones. Using devices planted on the same network allow for an opportunity for them to intercommunicate and work together to map and scan for vulnerabilities within a network. This would provide for maximum efficiency and faster vulnerability assessments. In the next section, we discuss the details of different load balancing scheme we designed.

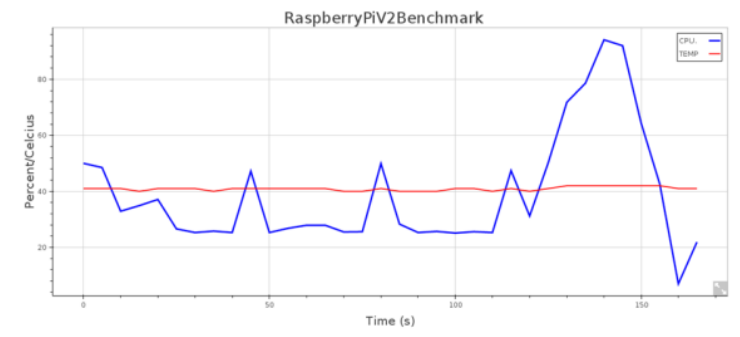

Figure 7. Vulnerability assessment benchmarking test

\section{Load balancing schemes}

Miniaturized computers, with their evercompetitive hardware, are great alternatives to the traditional and much more expensive desktop computing devices for vulnerability assessment purpose. Although these miniaturized computers can be utilized to perform tasks that previously require equipment with significant processing power for enterprise level vulnerability assessment, they are still outperformed in terms of most performance benchmarks. The key for improving assessment performance for large scale vulnerability assessment is to employ multiple miniaturized computers for the task and design an efficient load-balancing scheme. Load balancing is the process of evenly distributing workload across nodes in a network as to efficiently maximize resource potential and prevent overburdened workloads in individual nodes $[19,20$, 21]. Without load balancing, scanning a network with a significant number of nodes can take a huge toll on the miniaturized computer employed for the task. These scans can quickly exhaust the CPU reducing system performance and leading to poor average scan times [22]. On the other hand, if a load balancing method is not chosen carefully based on the target network environment and service load situation of individual nodes, simply adding more miniaturized computers is not a wise choice either. To maximize the utilization of the resource pool of multiple miniaturized computers for distributed penetration testing, a targeted load-balancing scheme has to be designed and implemented. We tested against baseline load balancing scheme with other possible solutions to determine which can provide the maximum resource utilization and performance.
All implementations proposed and tested are software based. Load balancing the scanning nodes, as discussed below, will lead to increased efficiency, faster scan durations, and overall system performance.

\subsection{Round robin scheme}

The most common and the simplest form of load balancing is round robin scheme. This made it a perfect test scheme for serving as the baseline for distributed penetration testing. Round robin load balancing is as easy as assigning IP addresses one by one to the miniaturized computers. Once the last miniaturized computer in the pool is assigned an IP address, the process restarts another round of IP address assignment. Each miniaturized computer will be responsible for scanning assigned nodes in the network. Although by default this simple scheme would not improve the efficiency of scanning any single node in the network for vulnerability assessment (since each node is only assigned to one miniaturized computer), it would balance utilization of the resource pool for scanning. Thus, the overall speed for vulnerability assessment for a computer network is dramatically increased by comparing scanning the network with a single miniaturized computer. This scheme is illustrated in Figure 8.

With a round robin implementation, the proof of concept for increased efficiency through load balancing is proven - but not substantial enough to leave as is. This leads us to propose multiple solutions to the problem of load balancing.

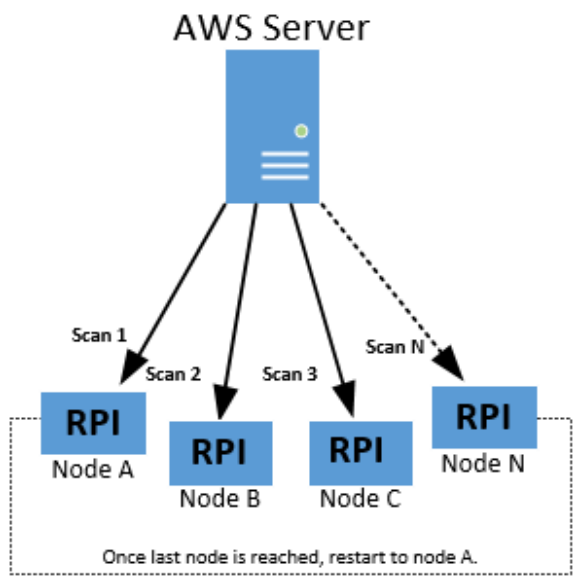

Figure 8. Round robin scheme for distributed penetration testing

\subsection{Dynamic load balancing}

Dynamic load-balancing provides a better alternative for creating a penetration testing architecture out of the miniaturized computer nodes for vulnerability assessment for networked services. This is because a simple round robin scheme assigns 
nodes to miniaturized computers without considering the loads on each miniaturized computer. So one miniaturized computer may finish work much earlier than some other ones. On the other hand, each individual computers to be scanned for vulnerabilities may have different number of services running there. Thus, it may take significant long time to scan a computer than others. We designed two dynamic load balancing schemes: load balancing based on CPU loads of penetration testing boxes and load balancing based on services running on individual computers to be scanned. We illustrate these two schemes as follows.

The dynamic load balancing scheme based on CPU loads of penetration testing boxes monitor changes on the miniaturized computers - such as current CPU usage - and factor these changes into the algorithm for distributing vulnerability assessment tasks among miniaturized computers in the resource pool. The proposed algorithm works as follows. At the beginning, each miniaturized computer is assigned one node for scanning. Then the vulnerability scanning process is started. Some miniaturized computers may finish vulnerability scanning earlier than others. Generally speaking, when the scanning is just started, the CPU load is high. Then it is lowered after certain amount of time. This can be seen from Figure 9 which depicts the CPU loads of 1-minute, 5-minute, and 15-minute duration of the scanning. Once the CPU load on one miniaturized computer drops below certain threshold, a new node in the network will be assigned to it for scanning. The process continues until all nodes in the network are scanned.

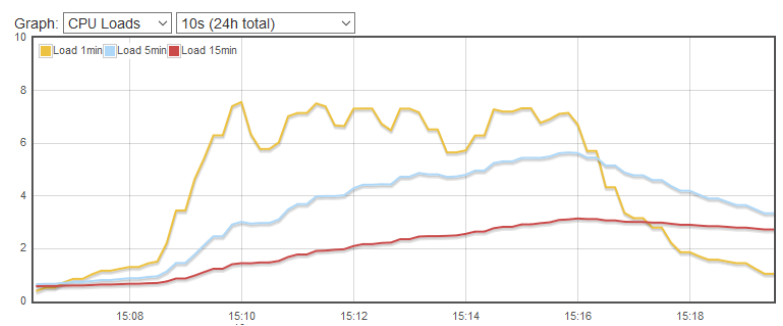

Figure 9. Penetration testing CPU load for different time durations

An example is as follows: miniaturized computer $\mathrm{M}$ and $\mathrm{N}$ are employed for scanning subnet $\mathrm{G}$ on a network. Subnet G contains 6 hosts, Host A, B, C, D, $\mathrm{E}$, and F. When an OpenVAS scan of the subnet is initiated from the AWS server, each host out of these 6 will be assigned either miniaturized computer $M$ or $\mathrm{N}$ for vulnerability scanning purpose. At the beginning, node $\mathrm{A}$ is assigned to $\mathrm{M}$ and node $\mathrm{B}$ is assigned to $\mathrm{N}$. Then $\mathrm{M}$ and $\mathrm{N}$ start their scanning processes. Usually there is a surge on CPU usage when the vulnerability scanning process just starts. After a certain amount of time, the CPU load starts to drop. Let us say, after 5 minutes, CPU load on the miniaturized computer $\mathrm{N}$ drops below the preset threshold. Then node $\mathrm{C}$ is assigned to $\mathrm{N}$ for vulnerability scanning also. Now $\mathrm{N}$ is responsible for finishing up scanning for node $\mathrm{B}$ and starting scanning node $\mathrm{C}$. This process continues until all 6 nodes are scanned for vulnerability assessment.

The dynamic load balancing scheme based on services running on individual computers to be scanned works as follows. First a port scanning is performed on the nodes in the network. This can be done by using the round robin scheme by assigning nodes to miniaturized computers. This step is similar to the steps illustrated in Section V.A. The only difference is that only port scanning is performed here. The port scanning process is used to identify the number of services running on individual nodes. This is because the more the number of services running on a node, the longer it takes to scan vulnerabilities on the node. After the port scanning step is finished, different number of miniaturized computers are assigned to each node in the network for vulnerability scanning. The assignment process is weighted based on the number of running services on a node. Multiple miniaturized computers assigned to the same node can perform the vulnerability scanning in parallel to speed up the process.

The benefits of dynamic load balancing scheme based either on CPU loads of penetration testing boxes or services running on nodes are increased efficiency and performance of vulnerability scanning process. The results from dynamic load balancing proved the advantage over the simple round robin scheme.

\subsection{Clustering Scheme}

The next option is clustering. There are resources out there on miniaturized computer clustering resulting in improved processing power [23, 24]. Cluster computing consists of multiple computing nodes interconnected so that they act as a single, more powerful, computing node [23, 24]. This type of computing scheme is actually used for supercomputers across the world. The idea behind this option is that if multiple miniaturized computers are scanning the same subnet, they can be clustered together to act as one, more powerful, node. This would increase the processing power of the new powerful "single-node" penetration testing box and again increase the overall performance of the system as a whole.

An example is as follows: There are 5 miniaturized computers used for vulnerability assessment for services running on a network. Miniaturized computer $\mathrm{M}, \mathrm{N}$, and $\mathrm{O}$ are employed for vulnerability scanning for subnet $\mathrm{A}$, while $\mathrm{P}$ and $\mathrm{Q}$ are utilized for vulnerability scanning for subnet B. When the AWS server determines that $\mathrm{M}, \mathrm{N}$, and $\mathrm{O}$ are in the same subnet on a network, a clustering 
command would be sent to the nodes, effectively combining them into one new "single-node", say, node MNO, which would now combine the resources of all individual nodes into one for undertaking vulnerability assessment tasks. The same would be done for $\mathrm{P}$ and $\mathrm{Q}$ on subnet $\mathrm{B}$, resulting in node $\mathrm{PQ}$. The advantage of employing clustering scheme is that clustering command will be able to simplify task scheduling for multiple miniaturized computers.

\subsection{Utilizing unused resources for vulnerability scanning}

This method could also be translated to adhere to the more popular distributed architecture described throughout this paper. As distributed computing projects, such as SETI@home [25], become more mainstream, the idea of distributed computing for combined processing power is becoming more viable for other computing tasks. In this instance we would create software that utilizes the unused resources of other nodes throughout the network for the scanning process of a target node. This option could be seen effectively as a scaled botnet used to maximize resource utilization towards vulnerability scanning.

One example for utilizing unused resources for vulnerability scanning is fairly simple. If we have node $\mathrm{M}, \mathrm{N}$, and $\mathrm{O}$ on a network, when a vulnerability scan is sent to node $\mathrm{M}$ and node $\mathrm{N}$ and $\mathrm{O}$ are idle, the system would utilize some of node $\mathrm{N}$ and O's processing power for scanning node $M$ for vulnerabilities. This would speed up scanning efficiency without adding extra computing equipment.

\section{Conclusions and future work}

The need for a safe computing environment will only grow with time. Projects like this provide alternatives to the expensive and resource intensive devices currently being used in the industry for vulnerability assessment and penetration testing. The design, implementation, and experiment of the project prove the viability of automated vulnerability assessment using OpenVAS and miniaturized computers. This paper outlines the methods for the project as well as the theory behind an automated distributed architecture for vulnerability assessment. After overcoming software and hardware incompatibilities, a boilerplate OS image for the Raspberry Pi devices was developed for use on new nodes and to help further enhance interest in the research. Multiple miniaturized computers can be plug into different networks and register themselves with our dashboard application which serves as the command center for performing vulnerability assessment on different networks.

Although penetration testing employing miniaturized computers is still in its early stage, systems utilizing these small low-power and low footprint devices may one day be the standard for IT security analysts worldwide.

This research was done with the intention of being continued, either by researchers or the open source community. Each process has a clear ending point where someone else can pick up and continue to work. Some of the top priority future endeavors are listed here:

- Optimize load balancing scheme for Raspberry Pi's on the same network for increased performance during network scans.

- $\quad$ Output more details about both the risk level of returned vulnerabilities and the priority in which they need to be taken care of on the dashboard.

- Allow users to choose the depth of their vulnerability scan.

\section{Acknowledgments}

This project is supported in part by a NKU Faculty Summer Fellowship, a NKU UR STEM project fund, and Greaves Undergraduate Summer Research Fellowship.

\section{References}

[1] OpenVAS, http://www.openvas.org

[2]Application Penetration Testing,www.trustwave.com/ apppentest.php

[3] E. U. Küçüksille, M. A. Yalçınkaya, S. Ganal, Developing a Penetration Test Methodology in Ensuring Router Security and Testing It in a Virtual Laboratory, in the Proceedings of the 8th International Conference on Security of Information and Networks, 2015.

[4] R. Li, D. Abendroth, X. Lin, Y. Guo, H. Baek, E. Eide, R. Ricci, J. Merwe, Potassium: Penetration Testing as a Service, in the Proceedings of the Sixth ACM Symposium on Cloud Computing, 2015.

[5] P. Tilak, White Paper on Penetration Testing, www.docstoc.com/docs/70280500/White-Paper-onPenetration-Testing.

[6] X. Qiu, Q. Jia, S. Wang, C. Xia, L. Lv, Automatic Generation Algorithm of Penetration Graph in Penetration Testing, in the Proceedings of the 2014 Ninth International Conference on P2P, Parallel, Grid, Cloud and Internet Computing, 2014.

[7] B. Stepien, L. Peyton, Innovation and Evolution in Integrated Web Application Testing with TTCN-3, International Journal on Software Tools for Technology Transfer (STTT), Volume 16, Issue 3, 2014. 
[8] M. Bishop, About Penetration Testing, IEEE Security \& Privacy, Volume 5, Issue 6, 2007.

[9] J. Muniz, Penetration testing with Raspberry Pi, Packt Publishing, 2015

[10] Pwn Plug, www.pwnieexpress.com. (Access date: 2 December 2015).

[11] MiniPwner, www.minipwner.com. (Access date: 7 December 2015)

[12] WiFi pineapple, www.wifipineapple.com. (Access date: 10 December 2015)

[13]Distributed Pentest Using Pi,github.com/ spinnyhatkid/PentestInABox

[14]Distributed Pentest Using Pi - Configuration and README, github.com/spinnyhatkid/PentestSSHConfigs

[15]Nessus, www.tenable.com/products/nessusvulnerability-scanner

[16] Nexpose, www.rapid7.com/products/nexpose/

[17] Core Impact, www.coresecurity.com/core-impact

[18] Canvas, www.immunityinc.com/products/canvas

[19] A. S. Milani and N. J. Navimipour, Load balancing mechanisms and techniques in the cloud environments: Systematic literature review and future trends, Journal of Network and Computer Applications, vol. 71, pp. 86-98, Jun. 2016.

[20] A. M. Alakeel, A Guide to Dynamic Load Balancing in Distributed Computer Systems, International Journal of Computer Science and Network Security, vol. 10, no. 6, pp. 153-160, Jun. 2010.

[21] A. Sharma and S. Verma, A Survey Report on Load Balancing Algorithm in Grid Computing Environment, International Journal of Advanced Engineering Research and Studies, pp. 128-132, Jan. 2015.

[22] A. T. Chronopoulos and S. Penmatsa, Dynamic MultiUser Load Balancing in Distributed Systems, In the proceedings of 2007 IEEE International Parallel and Distributed Processing Symposium, 2007.

[23] Z. Huang, H. Situ, and P. Werstein, Load Balancing in a Cluster Computer, in the proceedings of 2006 Seventh International Conference on Parallel and Distributed Computing, Applications and Technologies, 2006.

[24] J. Kiepert, RPi Cluster: Creating a Raspberry Pi-based Beowulf Cluster.

[25] Seti@home, https://setiathome.berkeley.edu/ 